\title{
Doubling effect of anti-microtubule herbicides on the maize haploid
}

\author{
Xuejiao Ren, Jiabin Ci, Xueyu Cui, Wei Yang* \\ Faculty of Agronomy, Jilin Agricultural University, Changchun, 130118, Jilin, China
}

\section{A B S T R A C T}

\begin{abstract}
Doubled haploid $(\mathrm{DH})$ technology is widely used in crop breeding programs. Colchicine is the most frequently used chemical agent in the $\mathrm{DH}$ inducing. However, colchicine has disadvantage of high poisonousness. It is necessary to find some non-toxic or low toxic substitutes that should have the same effect as colchicine. In this study, two anti-microtubule herbicides, amiprophosmethyl (AMP) and Oryzalin, were used to double the haploids under different treatment conditions, and the doubling effects were compared with colchicine. The results showed that the highest doubling rate induced by APM is about $45 \%$ in the condition of $20 \mu \mathrm{M} 24 \mathrm{~h}$. We calculated the actual doubling rate (survival rate $\times$ double rate), $38.23 \%, 20.64 \%$, and $19.4 \%$ are the highest actual doubling rates induced by APM, colchicine, and oryzalin, respectively. Although both APM and Oryzalin can induce maize haploid doubling, comparing with the actual doubling rate, APM is better than colchicine. In addition, soaking seed had been proved the best way to operate and obtain the highest doubling haploids in all conditions. As a low toxic mitotic inducer, APM is a good substitute of colchicine in doubling maize haploids, which is suitable for application in the $\mathrm{DH}$-based breeding pipeline.
\end{abstract}

Keywords: Maize; Anti-microtubule; Haploid; Doubling effect

\section{INTRODUCTION}

Haploid Plants, which contain only one complete set of chromosomes can be spontaneously or artificially, induced chromosome doubling to form "doubled haploids" (DH) (Arshadullah et al., 2018; Ong et al., 2017). In maize breeding, using DH technology breeders can shorten the breeding cycles and develop completely homozygous lines in 2-3 generations, compared with the conventional inbred line development process, which takes at least 6-8 generations to derive lines with $\sim 99 \%$ homozygosity (Geiger and Gordillo, 2009; Chang and Coe, 2009). Haploid induction has been recognized worldwide as an important measure for enhancing breeding efficiency and been adapted by commercial maize breeding programs, with maize haploid inducers available in breeding practice. (Schmidt, 2003; Seitz, 2005; Chen et al., 2009; Prigge and Melchinger, 2011; Kebede et al., 2011).

Chromosome doubling is necessary for maize haploids to restore fertility. Studies showed that colchicine can inhibit mitotic and induce chromosome doubling (Chase, 1969; Deimling et al., 1997). A scholar reported that 18\% of haploid seeds were chromosome doubled by treated $12 \mathrm{~h}$ with $0.06 \%$ colchicine (Davarnejad et al., 2018; Jiang et al., 2018; Khaleel et al., 2018; Sankarganesh et al., 2018). The fertility rate reached to $61 \%$ (Seaney, 1955) in roots treatment with colchicine. Although colchicine can induce chromosome doubling, it is not completely effective, because sectoral diploidization of inflorescences can occur. In addition, it has a disadvantage of high poisonousness (Stadler, 1989). Other herbicides, with less toxic (Geiger and Gordillo, 2009), such as pronamide, amiprophos methyl (APM), trifluralin, and Oryzalin, were also efficient mitotic inhibitors (Hantzschel and Weber, 2010). A scholar found that four different kinds of herbicide, APM, pronamide, Oryzalin and trifluralin, could cause chromosome doubling. Colchicine, Oryzalin and APM have similar function as colchicine by inhibiting the formation of microtubules in culture of anther of Brassica napus (Hansen et al., 1996). A scholar found that the doubling rate was about $11.05 \%$ by treated wheat with APM. APM treatment caused the mitotic index of onion meristem from $0.8 \%$ to $5.2 \%$ at mitotic metaphase (Zhen-ying, 2003).

\footnotetext{
*Corresponding author:

Xuejiao Ren, Agronomy college of Jilin Agricultural University, Changchun, 130118, Jilin, China. E-mail: davidyoung588@126.com
}

Revised: 14 January 2018; $\quad$ Accepted: 24 April 2018 
Anti-microtubule herbicides are lower toxic and more effective than colchicine in chromosome doubling in plant (Li et al., 2018; Wang et al., 2018). However, few studies are about haploid doubling by treated with antimicrotubule herbicides in maize. In order to choose a low toxic and effective haploid doubling reagent, two kinds of anti-microtubules, AMP and Oryzalin, and colchicine were used to treat seeds, buds, and shoots of maize haploids in different concentrations at different growth time points (Howlader et al., 2018; H'ng et al., 2018; Fahim and Sathi, 2018). Moreover, the results of this study may provide the theory basis and feasible measure for improving the technology of haploid doubling in maize.

\section{MATERIALS AND METHODS}

Maize haploids used in this research were induced from a hybrid (Zheng $58 \times$ PH6WC) by the haploid inducer, JS6-2. We set three treatment groups, the seed immersion group, the bud immersion group, and the root immersion group. In each treatment, 50 putative haploid progenies were treated and planted at the Breeding Station of Jilin agricultural university, Changchun, China. The stock solution of APM or Oryzalin is $3 \mathrm{mM}$, which were mixed with $0.1 \%$ Tween- 20 and $2 \%$ DMSO. Final concentrations $(10 \mu \mathrm{M}, 15 \mu \mathrm{M}, 20 \mu \mathrm{M}, 25 \mu \mathrm{M}$, and $30 \mu \mathrm{M})$ were adjusted by ddH2O. Colchicine with the concentration of $0.5 \mathrm{mM}$, $1.0 \mathrm{mM}, 1.5 \mathrm{mM}$, and $2.0 \mathrm{mM}$ were prepared in $0.1 \%$ Tween-20 and 2\% DMSO and dissolved in ddH2O, respectively. For the seed immersion, the haploid seeds were soaked in water for $6 \mathrm{hrs}$ at room temperature, and then cut slightly around the embryo. The cut seeds were immersed in the different agents (AMP, Oryzalin, or Colchicine) at $28^{\circ}$ for $8 \mathrm{~h}, 16 \mathrm{~h}, 24 \mathrm{~h}$, and $48 \mathrm{~h}$. Where after they were rinsed with water for 30 minutes. For the bud immersion, the seeds were soaked in water at room temperature for $6 \mathrm{hrs}$. and germinated on a wet germination paper at $28^{\circ}$. After the roots had grown to about $1 \mathrm{~cm}$, the temperature was lowered to $24^{\circ}$ in order to make the buds to germinate. When the shoots grew to about $2 \mathrm{~cm}$, we cut the top $3 \mathrm{~mm}$ of the coleoptiles, which were soaked at $28^{\circ}$ in the treatment agents for $8 \mathrm{~h}, 16 \mathrm{~h}, 24 \mathrm{~h}$, and $48 \mathrm{~h}$. Later they were soaked in water for $30 \mathrm{~min}$ and then grown in nursery site at greenhouse $\left(28^{\circ}\right)$ before they grew to 4 or 5 leaf-age which can be transplanted to field. For the root immersion, the seeds were soaked in water at room temperature for 6 hrs. and placed in an incubator at $28^{\circ}$ until the roots grew up to $2 \mathrm{~cm}$. They were placed in the $4-8^{\circ}$ condition for $3 \mathrm{hrs}$. Then, the roots were immersed in the agents he treatment agents for $8 \mathrm{~h}, 16 \mathrm{~h}, 24 \mathrm{~h}$, and $48 \mathrm{~h}$ before transplanted to the field. All three treatments were replicated 3 times, respectively.
The chromosome ploidy level of induced progenies was checked by Flow cytometry (FCM). When the third leaf emerged, the tip of second leaf was cut off, chopped into small pieces and stained with $2 \mathrm{ml}$ DAPI (4'6-diamidino-2-phenylindone) solution $(5 \mu \mathrm{g} / \mathrm{ml}$, Partec $\mathrm{GmbH}$, Germany), filtered through a nylon membrane (50 $\mu \mathrm{m}$ mesh size). The filtrate was analyzed by FCM, at a par gain FL1 (fluorescence) of 420-430 nm (relative fluorescence-RF). The peak of DH should be 60-130 FL. In contrast, the peak of haploid plant should be 30-70 FL (Dang et al., 2012).

\section{RESULTS}

In order to compare the doubling effect of haploids by using different ways, the survival rate and doubling rate of haploids were analyzed. Under the three different chemical treatments, the results of survival rate of haploids showed that survival rates obviously decreased with the increase of the concentration and the processing time in the most kinds of treatment groups (Fig 1). Especially, in each groups of haploid seeds treated by APM, the survival rates were not significant different, which indicated the toxic effects of APM to haploid seeds was limited and not obviously enhancing with the concentration increasing.

Comparing the different approaches, the survival rate in the seed-soaked group was significantly higher than the others. This result indicated that root tips and bud tips were more sensitive to the chemical treatments. And the mortality rates increased significantly with the concentration increasing in the bud/root treated groups. Soaking seeds is not only easy to operate but also suitable to get more survival seedlings, which is better than soaking bud/root ways.

To compare the doubling effect of three chemicals, the double rates have been analyzed. The results showed that APM was better than the other two (Fig 2). The highest doubling rate induced by colchicine is about $30 \%$ in the condition of $1.5 \mathrm{mM} 24 \mathrm{~h}$; the highest doubling rate induced by APM is about $45 \%$ in the condition of $20 \mu \mathrm{M} 24 \mathrm{~h}$; and highest doubling rate induced by oryzalin is about $32 \%$ in the condition of $25 \mu \mathrm{M} 24 \mathrm{~h}$. We calculated the actual doubling rate (survival rate $\times$ double rate), $38.23 \%, 20.64 \%$, and $19.4 \%$ are the highest actual doubling rates induced by APM, colchicine, and oryzalin, respectively. We also found that all the highest doubling rates in our research were induced in the soaking seed groups.

Comprehensively analyzing various Doubling methods based on the average value of haploid doubling rates, we 


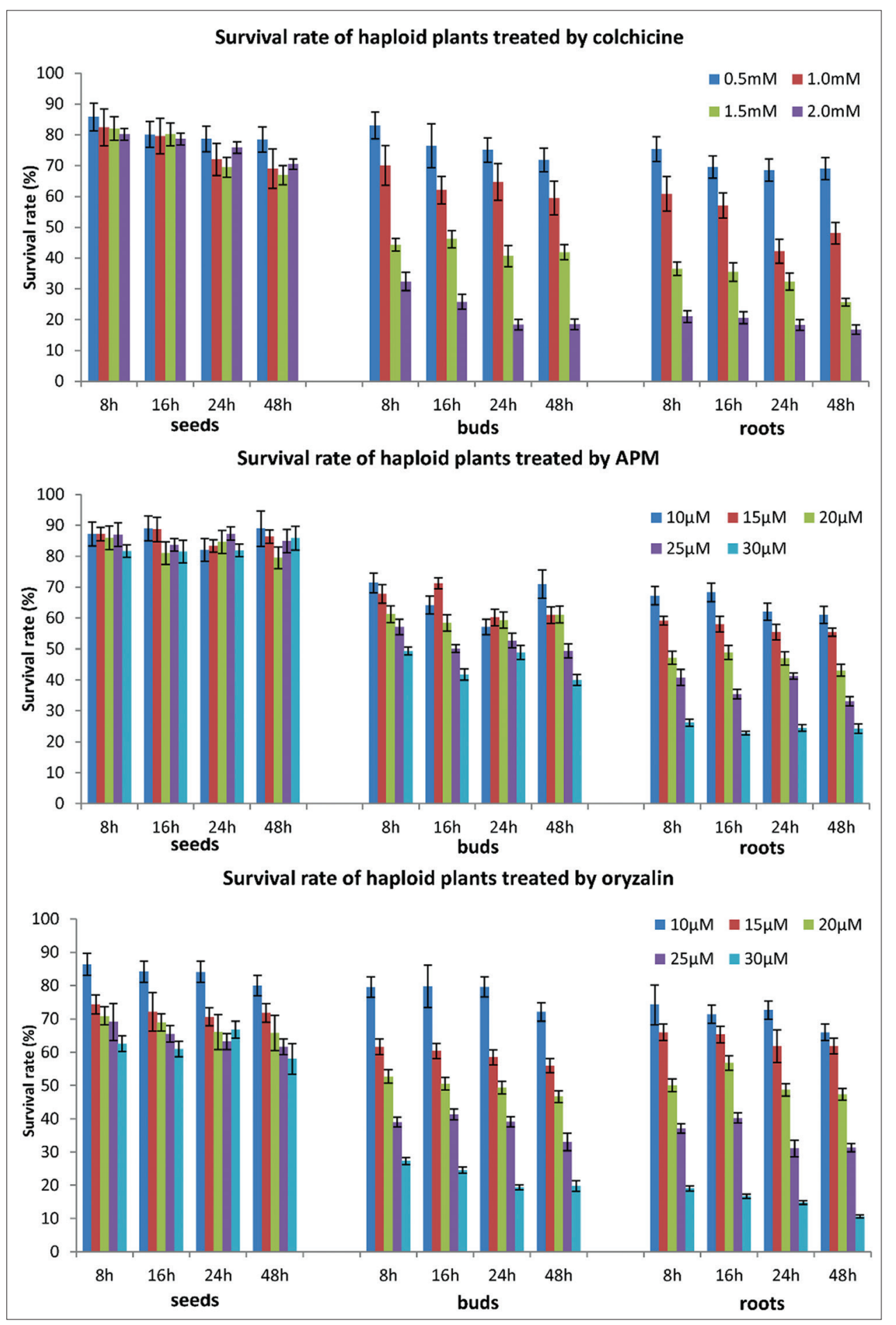

Fig 1. Survival rate of maize haploids treated by colchicine, APM, and oryzalin.

clearly found, APM is the best to induce haploid doubling in the three kinds of agents, while soaking seed not only has the advantage of easy operation, but also had obtained the significantly higher doubling effect than the other ways (Table 1). In this study, the best haploid doubling way was the haploid seeds were soaked in the $20 \mu \mathrm{M}$ of APM for $24 \mathrm{~h}$. Using colchicine, the best way was to soak seeds in the $1.5 \mathrm{mM}$ of colchicine for $24 \mathrm{~h}$; Using ORYZALIN, the best way was to soak seeds in the $25 \mu \mathrm{M}$ of ORYZALIN for $24 \mathrm{~h}$.

\section{DISCUSSION}

Many studies have proved that the maize lines which come from haploid doubling are completely homozygous and homogeneous. Doubled haploid lines have the 


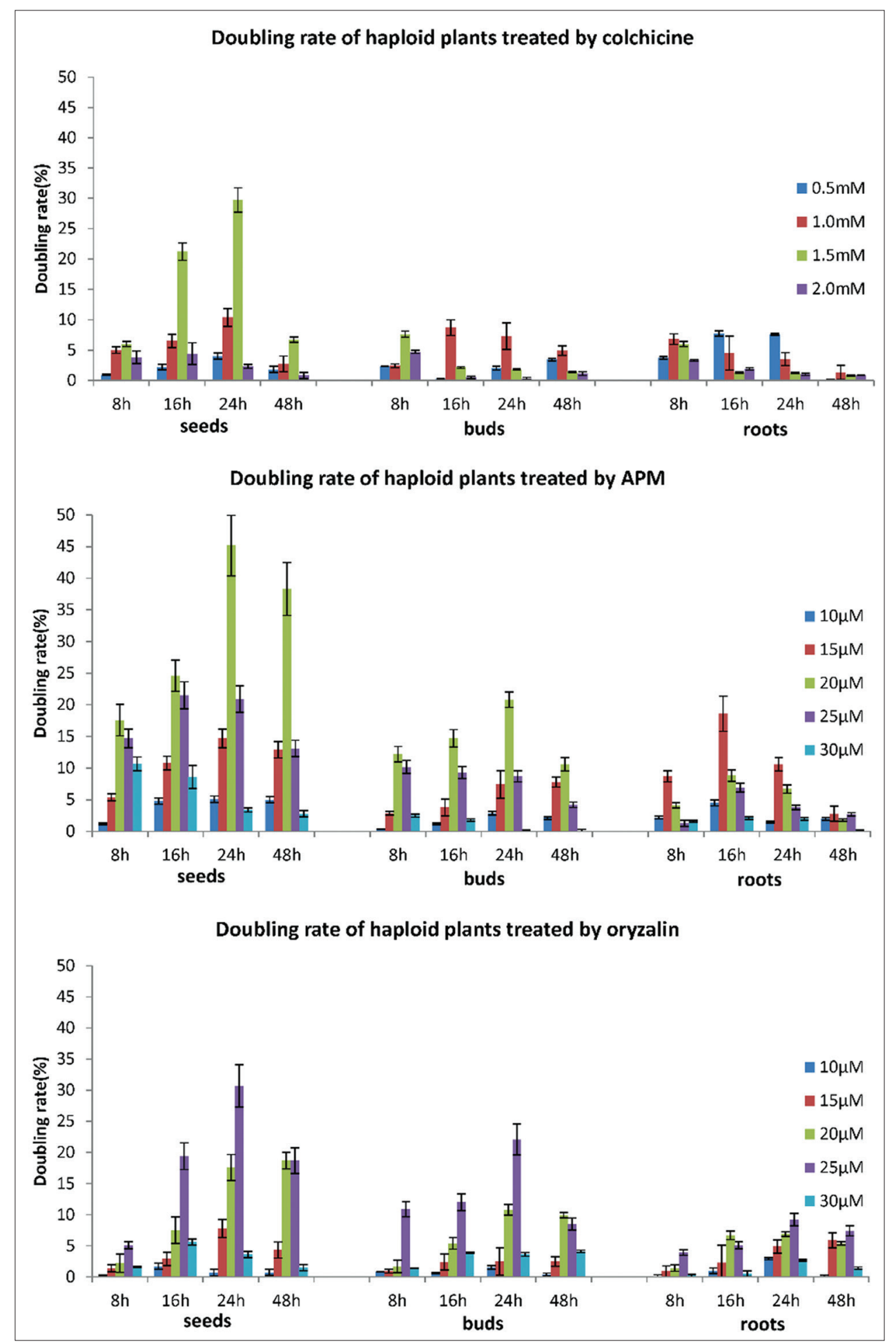

Fig 2. Doubling rate of maize haploids treated by colchicine, APM, and oryzalin.

advantage of increasing the efficiency of selection, reducing the breeding cycles, and decreacing the effort for line maintenance (Chang and Coe, 2009; Geiger and Gordillo, 2009; Röber et al., 2005; Geiger, 2009). Our studies showed that the spontaneous doubling rate was very low at only about $1.5 \%$. Colchicine is most frequently used for inducing haploid doubling. In this study, the highest doubling rate of colchicine was $29.7 \%$, which was remarkably higher than the spontaneous haploid doubling rate. Other studies on colchicine were similar to ours. The highest doubling rate of colchicine treatment in Wen's study was $48.35 \%$, while a scholar's results showed that the highest doubling rate was about 23\% (Liu, 2000). Another study showed that a haploid doubling rate of $19.8 \%$ appeared with treatment in six-leaf-stage by colchicine (Wei, 2007).

Emir. J. Food Agric • Vol 30 •Issue $10 \bullet 2018$ 
Table 1: Comparison of the doubling effect under the different treatments

\begin{tabular}{lcccccc}
\hline & \multicolumn{7}{c}{ Doubling rate } \\
\cline { 2 - 7 } & \multicolumn{2}{c}{ Seed soaked } & \multicolumn{2}{c}{ Bud soaked } & \multicolumn{2}{c}{ Root soaked } \\
\hline Colchicine & $5.10 \%$ & $\mathrm{Ba}$ & $1.77 \%$ & $\mathrm{Cb}$ & $1.68 \%$ & $\mathrm{Bb}$ \\
APM & $11.82 \%$ & $\mathrm{Aa}$ & $3.60 \%$ & $\mathrm{Ab}$ & $2.35 \%$ & $\mathrm{Ac}$ \\
Oryzalin & $5.00 \%$ & $\mathrm{Ba}$ & $2.23 \%$ & $\mathrm{Bb}$ & $1.58 \%$ & $\mathrm{Bc}$
\end{tabular}

$A, B, C$ present the significant difference between the agents; $a, b, c$ presents the significant difference between the treated organs $(P<0.01)$

Although colchicine was used to induce haploid doubling widely, it has disadvantage of high poisonousness (Stadler, 1989). It is necessary to find some non-toxic or low toxic substitutes that should have the same effect as colchicine. Some herbicides, such as pronamid, amiprophosmethyl (APM), trifluralin, and Oryzalin, are good candidates, because they are not only efficient mitotic inhibitors but also less toxic compared to colchicine (Geiger and Gordillo, 2009; Hantzschel and Weber, 2010). In our study, APM and Oryzalin were selected to research the effect of inducing haploid doubling in maize.

Compared with Oryzalin and colchicine, the survival rate under APM treatment was higher. The results indicated that the inhibitory action of APM was weaker than oryzalin and colchicine in maize seedling development. A previous study found that APM can effectively double the chromosomes of callus tissue with no toxic effect on regenerated plants (Wan et al.1991). Similar results also showed that APM did not inhibit "Black Mexican Sweet" callus culture at $50 \mu \mathrm{M}$ for 21-28 hrs. Oryzalin also has an effect on doubling haploid. Some researchers found that callus regeneration was decreased severely at high concentration of Oryzalin, while a low concentration did not double chromosome efficiently (Duncan and Widholm, 1989). In our study, Oryzalin treatment was found to have a lower survival rate compared with the other two methods, although the doubling rate was better than colchicine.

Our results showed that both APM and Oryzalin can induce maize haploid doubling. However, comparing the actual doubling rate, just APM is better than colchicine. In addition, soaking seed had been proved to be the best way to operate and obtain the highest doubling haploids in all conditions. In this stage, a large number of cells in haploid seeds are in the period of mitosis, which makes them more likely to be treated by chemical agents.

We observed the morphological changes of the haploid seeds throughout the treatment process. No growth was observed during the process of soaking seed. In the recovery process, the soaked seeds resumed normal growth, and SAM (shoot apical meristems) regions were observed in most of soaked seeds (Supplementary Fig 1). The same phenomenon was reported in a previous study (Hantzschel et al., 2010).

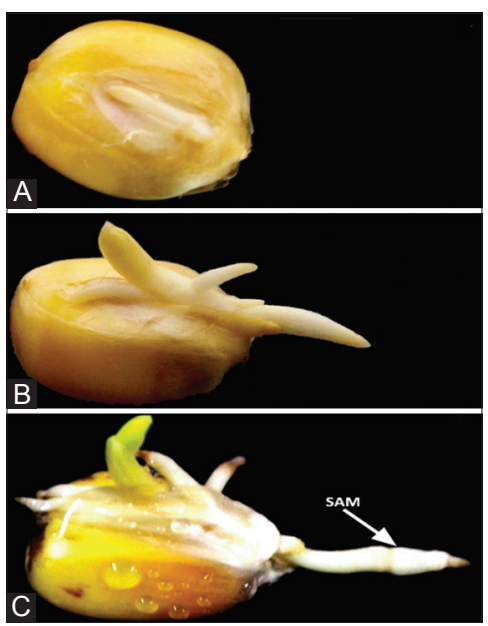

Supplementary Fig 1. Examples of morphological changes in the treatment A, a seed soaked. B, a seed in the growth recovery; C, a $S A M$ region in the soaked seed.

In conclusion, in all methods of doubling haploids, soaking seeds with APM showed that the best results, although all three chemical agents, APM, Oryzalin, and colchicine, have the effect of inducing haploid doubling. In our research, the highest doubling rate induced by APM is about $45 \%$ in the condition of $20 \mu \mathrm{M} 24 \mathrm{~h}$. As a low toxic mitotic inhibitor, APM is a good substitute to colchicine in doubling maize haploids, which is suitable for application in the $\mathrm{DH}$ based breeding pipeline.

\section{ACKNOWLEDGMENTS}

This work was supported by the National Science and Technology Major Project (2014ZX800305B), National Science \& Technology Pillar Program (2014BAD01B01) and Agriculture Science Technology Achievement Transformation Fund (2014GB2B100006); The National Key Research and Development Program of China (2017YFD0101102).

\section{REFERENCES}

Alan, A. R., W. Lim, M. A. Mutschler and E. D. Earle. 2007. Complementary strategies for ploidy manipulations in gynogenic onion (Allium cepa L.). Plant Sci. 173(1): 25-31.

Arshadullah, M., M. Suhaib, R. Baber, M. Usama, B. uz-Zaman, I. A. Mahmood and S. I. Hyder. 2017. Growth of Chenopodium quiona wild under naturally salt affected soils. Malays. J. Sustain. Agric. 1(1): 1-3.

Chang, M. T. and E. H. Coe. 2009. Biotechnology in Agriculture and Forestry. Vol. 63. Molecular Genetic Approaches to Maize Improvement, Springer Verlag., Berlin, Heidelberg, pp. 127-142.

Chang, M. T. and E. H. Coe. 2009. Doubled haploids. In: Kriz, A. L. and B. A. Larkins (Eds), Biotechnology in Agriculture and Forestry. Vol. 63. Molecular Genetic Approaches to Maize Improvement, Springer Verlag., Berlin, Heidelberg. pp. 127-142.

Chase, S. S. 1969. Monoploids and monoploid-derivatives in maize (Zea mays L.). Bot. Rev. 35: 117-167. 
Chen. S., L. Li and H. Li. 2009. Maize Doubled Haploid Breeding [In Chinese]. China Agricultural University Press., Beijing.

Davarnejad, R., M. K. Moraveji and M. Havaie. 2018. Integral technique for evaluation and optimization of $\mathrm{Ni}$ (ii) ions adsorption onto regenerated cellulose using response surface methodology. Arabian J. Chem. 11(3): 370-379.

Deimling, S., F. K. Rober and H. H. Geiger. 1997. Methodology and genetics of in vivo haploid induction in maize [In German]. Vortr Pflanzenz üchtg. 38: 203-224.

Duncan, D. R. and J. M. Widholm. 1989. Differential response to potassium permanganate of regenerable and of non-regenerable tissue cell walls from maize callus cultures. Plant Sci. 61: 9I-103.

Fahim, N. F. and Z. S. Sathi. 2018. Assesment of hepatoprotective activity of roots and barks of Achyranthes aspera in carbon tetrachloride-induced hepatotoxicity in rats. Malays. J. Halal Res. 1(2): 23-26.

Gayen, P. and K. R. Sarkar. 1996. Cytomixis in maize haploids. Indian J. Genet. Plant Breed. 56: 79-85.

Geiger, H. H. and G. A. Gordillo. 2009. Doubled haploids in hybrid maize breeding. Maydica. 54: 485-499.

H'ng, S. Y., M. S. Anuar and M. Z. M. Nor. 2018. Drying, colour and sensory characteristics of 'berangan' banana (Musa accuminata) flesh dried using a microwave oven. Malays. J. Halal Res. 1(1): 10-14

Hansen, N. J. P. and S. B. Andersen. 1996. In vitro Chromosome Doubling Potential of Colchicine, Oryzalin, Trifluralin, and APM in Brassica napus Microspore Culture. Vol. 88. Euphytica, Kluwer Academic Publishers., Printed in the Netherlands. pp. 159-164.

Hantzschel, K. R. and G. Weber. 2010. Blockage of mitosis in maize root tips using colchicine-alternatives. Protoplasma. 241: 99-104.

Howlader, M. H. K., M. N. Islam, S. Biswas, M. E. Uddin, A. Shila, M. Z. Haque and N. Mahmud. 2018. Salt tolerance of chili genotypes during germination and seedling growth. Malays. J. Halal Res. 1(2): 1-7.

Islam, S. M. S. 2010. The effect of colchicine pretreatment on isolated microspore culture of wheat (Triticum aestivum L.). Aust. J. Crop Sci. 4(9): 660-665.

Jiang, M., H. Wu, Z. Li, D. Ji, W. Li, Y. Liu, D. Yuan, B. Wang and Z. Zhang. 2018. Highly selective photoelectrochemical conversion of carbon dioxide to formic acid. ACS Sustain. Chem. Eng. 6(1): 82-87.

Kebede, A. Z., B. S. Dhillon, W. Schipprack, J. L. Araus, M. Banziger, K. Semagan, G. Alvarado and A. E. Melchinger. 2011. Effect of source germplasm and season on the in vivo haploid induction rate in tropical maize. Euphytica. 180: 219-226.
Khaleel, C., N. Tabanca and G. Buchbauer. 2018. Alpha-terpineol, a natural monoterpene: A review of its biological properties. Open Chem. 16(1): 349-361.

Li, Z., C. Han and D. Wei. 2018. Empirical research on the relationship between natural gas consumption and economic growth in the northeast Asia. Energy Environ. 29(2): 216-231.

Dang, N. C. and M. Munsch. 2012. Inducer line generated double haploid seeds for combined waxy and opaque 2 grain quality in subtropical maize. Euphytica. 183: 153-160.

Ong, S. Q., B. B. Lee, G. P. Tan and S. Maniam. 2017. Capacity of black soldier fly and house fly larvae in treating the wasted rice in Malaysia. Malays. J. Sustain. Agric. 1(1): 8-10.

Prigge, V. and A. E. Melchinger. 2011. Production of haploids and doubled haploids in maize. Plant cell culture protocols, $3^{\text {rd }} \mathrm{Ed}$. Humana Press-Springer Verlag., Totowa, New Jersey.

Sankarganesh, M., J. Rajesh, G. G. V. Kumar, M. Vadivel, L. Mitu, R. S. Kumar and J. D. Raja. 2018. Synthesis, spectral characterization, theoretical, antimicrobial, DNA interaction and in vitro anticancer studies of $\mathrm{Cu}$ (ii) and $\mathrm{Zn}$ (ii) complexes with pyrimidine-morpholine based schiff base ligand. J. Saudi Chem. Soc. 22(4): 416-426.

Schmidt, W. 2003. Hybrid maize breeding at KWS SAAT AG. In: Bericht über die Arbeitstagung der Vereinigung der Pflanzenz üchter und Saatgutkaufleute Oerreichs, Gumpenstein., Oterreich. pp. 25-27.

Seaney, R. R. 1955. Studies on Monoploidy in Maize Ph.D. Thesis Cornell University, Ithaca., New York.

Seitz, G. 2005. The use of doubled haploids in corn breeding. In: Proc. 41 $1^{\text {st }}$ Annual Illinois Corn Breeders' School 2005, Urbana Champaign., Illinois. pp. 1-7.

Stadler, J., R. Phillips and M. Leonard. 1989. Mitotic blocking agents for suspension cultures of maize black Mexican sweet cell lines. Genome. 32: 475-478.

Wan, Y., D. R. Duncan and A. L. Rayburn. 1991. The use of antimicrotubule herbicides for the production of doubled haploid plants from anther-derived maize callus. Theor. Appl. Genet. 81(2): 205-211

Wang, M., D. Q. Zhang, J. Su, J. W. Dong and S. K. Tan. 2018. Assessing hydrological effects and performance of low impact development practices based on future scenarios modeling. J. Clea. Prod. 179: 12-23.

Zhen-ying, W., L. G. Cheng and H. Cheng. 2003. Induction of abnormal mitosis and change in protein compositions after treatment of root meristerms with phosphoric amide her bicide APM in Allium. Acta Agron. Sin. 29(4): 545-550. 